\title{
DISTÚRBIOS DO SONO EM PACIENTES IDOSOS COM DEMÊNCIAS
}

\author{
Mariana de Lemos Santos de Souza Oliveira ${ }^{1}$ \\ Alcione Silva de Carvalho
}

RESUMO: Os Distúrbios do Sono estão cada vez mais frequentes na vida da população devido a diversos fatores do dia a dia. Com o aumento da expectativa de vida, nota-se que especificamente os idosos são um grupo que sofre bastante com essas perturbações do sono. Atrelado a isto temos as condições físicas e psicológicas de idosos além de doenças como as Demências que diminuem as chances de se ter uma boa qualidade de sono. Importante ressaltar que idosos recorrem a algumas medicações e estas podem ser também gatilhos para que não haja um bom sono reparador. Dessa forma o Farmacêutico exerce um papel especial ao informar sobre os medicamentos e seus efeitos colaterais sobre o sono de idosos com demências, além disso, traz soluções hábeis para se obter uma boa noite de sono.

Palavras- Chave: Demências. Distúrbios do Sono. Idosos.

ABSTRACT: Sleep Disorders are more and more frequent in the population's life due to several factors of daily life. With the increase in life expectancy, it is noted that specifically the elderly are a group that suffers a lot from these sleep disturbances. Linked to this are the physical and psychological conditions of the elderly, as well as diseases such as Dementias that reduce the chances of having a good quality of sleep. Importantly, elderly people use some medications and these can also be triggers for not having good restful sleep. In this way, the Pharmacist plays a special role in informing about medications and their side effects on the sleep of elderly people with Dementia, and also brings skillful solutions to obtain a good night's sleep.

Keywords: Dementias. Sleep Disorders. Seniors.

\section{INTRODUÇÃO}

A qualidade do sono é primordial pra se ter uma vida saudável seja no âmbito físico, social, mental e cognitivo. Com o aumento da expectativa de vida dos brasileiros se tornou cada vez mais atraente descobrir tratamentos e medicações por parte da indústria farmacêutica pra que se

\footnotetext{
I Graduação em Farmácia. Universidade Iguaçu - Faculdade de Ciências Biológicas e da Saúde. Email: maris.lemos96@gmail.com.

${ }^{2}$ Doutora em Ciências e Mestre em Ciências pelo Instituto de Química da Universidade Federal do Rio de Janeiro (IQ-UFRJ). Docente e Orientadora no curso de graduação em Farmácia na Universidade Iguaçu.

E-mail: alcionecarvalhor57o@gmail.com.
} 
obtenha condições de ter uma velhice proveitosa. No entanto acontece com a maioria das pessoas durante o processo de envelhecimento o aparecimento de doenças crônicas e quadros de Demências. Este acarreta modificações qualitativas e quantitativas do sono.

Por isso é necessário entender o termo Demência como uma "síndrome da alteração da memória e da cognição acompanhada de declínio no funcionamento social e ocupacional estando, no entanto, conservados os níveis de consciência e de alerta." (i.e.,sensorium) (Andersen N. et Al 2019).

A Demência é uma síndrome naturalmente adquirida e difícil de ser compreendida dependendo do indivíduo em questão, ou seja,da área que o cérebro é afetado. Dessa forma não é tão fácil encontrar a origem e também o tratamento específico porém é necessário com o aumento da existência de vida saber lidar com a situação e descobrir metodologia específica que funcione pra dar uma vida mais saudável. Para isto, o farmacêutico deve possuir o conhecimento teórico, aliado à habilidade de comunicação nas relações interpessoais (Chaud, Gremião, Freitas, 2008). Assim, será possível analisar os distúrbios do sono e buscar encontrar soluções tanto pra idosos como para a geração jovem de hoje pois poderão ter um sono agradeço e saberão lidar com problemas (Demências e outros transtornos) que talvez venham futuramente.

\section{OBJETIVO GERAL}

Este trabalho tem como objetivo mapear a incidência de distúrbios do sono em pacientes idosos com Demências de acordo com o processo de envelhecimento progressivo, acompanhamento e métodos utilizados para benefício do paciente, a importância da orientação para que o paciente idoso tenha uma Farmacoterapia e qualidade de sono eficaz.

\section{OBJETIVOS ESPECÍFICOS}

- Analisar a existência de distúrbios do sono em pacientes idosos que apresentem quadro clínico de Demências;

- Acompanhamento de fatores que influenciem no sono de idosos como por exemplo ritmo Circadiano, comorbidades existentes, medicamentos utilizados de forma contínua e também o psicológico e físico de cada indivíduo;

- Traçar o perfil sócio econômico e doenças crônicas existentes que porventura possam atrapalhar a dinâmica do sono; 
- Relacionar o sono de idosos da atualidade por meio de pesquisas com a expectativa de vida que cada vez mais está elevando de forma que haja melhoria e assim consequentemente haja benefícios de qualidade de sono e portanto qualidade de vida também;

- Esclarecer dúvidas em relação a administração dos fármacos em idosos com Demências;

- Orientar os pacientes e cuidadores sobre possíveis intervenções medicamentosas em relação a qualidade do sono;

- Informar os riscos de se ter uma má qualidade de sono pra atividades rotineiras e ensinar soluções baseadas em pesquisas que tragam vantagens para a vida de idosos, cuidadores e população em geral.

\section{METODOLOGIA}

O trabalho exposto foi realizado com uma pesquisa bibliográfica onde foi possível verificar os ensinamentos de autores que por vezes já vinham discutindo acerca do tema abordado, dentre outros que esboçam sua preocupação com a qualidade de sono de idosos com Demências assim como da população em geral com a expectativa de vida; os medicamentos utilizados e assim acredita ser relevante para a discussão com o intuito de auxiliar outros trabalhos e vislumbrar mais informações acerca do tema.

A busca do material bibliográfico deu-se nos seguintes documentos aplicado através da busca de materiais de dados eletrônicos, a saber: Google Acadêmico (pesquisa avançada) e Scientific Eletronic Library Online - SciELO, e Pubmed, e livros, que relata os assuntos em questão, os quais contribuíram a elevar a melhor compreensão do tema a ser apresentado neste trabalho.

\section{JUSTIFICATIVA}

A reflexão acerca do período de Sono especificamente em indivíduos idosos remete a um paralelo sobre a qualidade psicológica, física e social que a maturidade com o passar dos anos trás. A Demência atinge a maior parte dos pacientes com idade avançada e o padrão do sono é totalmente afetado.

Através do Ciclo Circadiano consegue-se definir os estágios do sono para que haja descanso mental e reposição energética para um novo dia porém isso não ocorre em pessoas com alguma problemática referente a demências. O sono diurno passa a ser inevitável e o noturno passa a ser 
de vigilância, quando não se tem repouso efetivo. Dessa forma através de pesquisas esse trabalho tem o intuito de mostrar um roteiro idade $\mathrm{x}$ Demências $\mathrm{x}$ aspectos externos mostrando a relevância de se ter qualidade de sono pra exercer as atividades diárias com sucesso.

Além disso, apontar o papel do Farmacêutico como profissional de saúde que esclarece dúvidas sobre a influência de medicamentos e o sono tanto pra idosos quanto pra população em geral que também chegará nessa idade futuramente com o aumento da expectativa de vida e assim deve-se estudar o processo pra que compreenda-se os prós e contras que a idade trás sobre o sono e solucioná-lo pra uma melhor qualidade de vida futura.

\section{DESENVOLVIMENTO}

A proporção de idosos na população brasileira vem crescendo rapidamente desde o início da década de 1960, quando a queda das taxas de fecundidade e o aumento da expectativa de vida começaram a alterar sua estrutura, estreitando progressivamente a base da pirâmide populacional e alargando seu ápice, o que ocasiona redefinição nas responsabilidades familiares e nas demandas por políticas públicas, alterando até mesmo as relações de gênero no seio familiar.

Estima-se que, em 2025, a população brasileira terá um aumento de cinco vezes em relação à de 1950, ao passo que o número de pessoas com idade superior a 60 anos terá aumentado aproximadamente is vezes. Como conseqüência, o Brasil assumirá a sexta posição entre os países mais envelhecidos do mundo. Em geral, com o aumento da idade cronológica, ocorre uma maior prevalência de condições crônicas de saúde, o que predispõe os idosos a um maior consumo de medicamentos. Vale ressaltar que esse grupo etário apresenta peculiaridades em relação à utilização da farmacoterapia, se comparado ao restante da população.

A Atenção Farmacêutica (AtenFar), prática desenvolvida no contexto da assistência farmacêutica, tem aumentado muito nos últimos anos e sua consolidação tem sido gradativa, sobretudo nos países em desenvolvimento. O objetivo da AtenFar não é intervir no diagnóstico ou na prescrição de medicamentos, atribuições do médico, mas garantir uma farmacoterapia racional, segura e custo-efetiva. (Meneses, Barreto Sá, 2007). 


\section{ORGANIZAC̨ÃO MUNDIAL DE SAÚDE}

Mais da metade da população mundial de Idosos (maiores de 60 anos), em expansão, está nos países em desenvolvimento - em milhões de Idosos

HOJE 600 Milhões

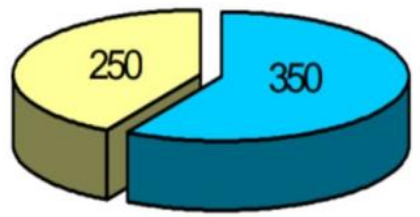

Países em vias de desenvolvimento
EM 2020 Mais de 1000 Milhões

\section{0}

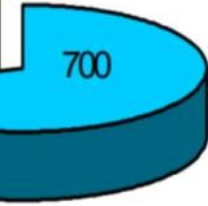

Países desenvolvidos

\section{GRÁFICO 1- Percentagem de idosos face à população mundial}

Fonte: A Perspectiva do Farmacêutico frente a doença de Alzheimer

\section{DISTÚRBIOS DO SONO EM IDOSOS}

Dessa forma com o aumento da longevidade entende-se que algumas problemáticas podem ocorrer e uma delas em questão são os distúrbios do sono em pacientes idosos. Na população em geral os principais distúrbios do sono são Insônia, apneia obstrutiva do sono e síndrome das pernas inquietas porém a arquitetura do sono em idosos sofre mudanças devido a estrutura física que não é a mesma com o passar dos anos. Diminuição nas funções renais, cardíacas e cerebrais alteram o sono.

Ocorre modificações como diminuição no tempo de sono, geralmente os idosos ficam em estado de "alerta", demoram a "pegar" no sono, dormem pouco a noite e tem vários cochilos durante o dia. SED significa Sonolência Excessiva Diurna e é relacionada com o Ritmo Circadiano que é o mecanismo pelo qual nosso organismo se organiza entre o dia e a noite.

A partir dele, todos os processos fisiológicos são comandados para que o corpo consiga acordar, sentir fome, estar ativo, ficar com sono entre outros. Porém com o passar dos anos o corpo do idoso sofre alterações e não é mais o mesmo, dessa forma o idoso indo dormir mais cedo tem o despertar bem antes e também tem o sono-vigilia aumentado, ficando em alerta.

Assim, o idoso passa a tirar vários cochilos durante o dia e dormir pouco a noite, não sendo saudável pois a secreção endógena de melatonina é diminuída com aumento da idade. O sono é 
mais vago (aumento das fases I e II e diminuição das fases de ondas lentas) e mais curto (diminuição do tempo de sono).

Figura: Ciclo Circadiano

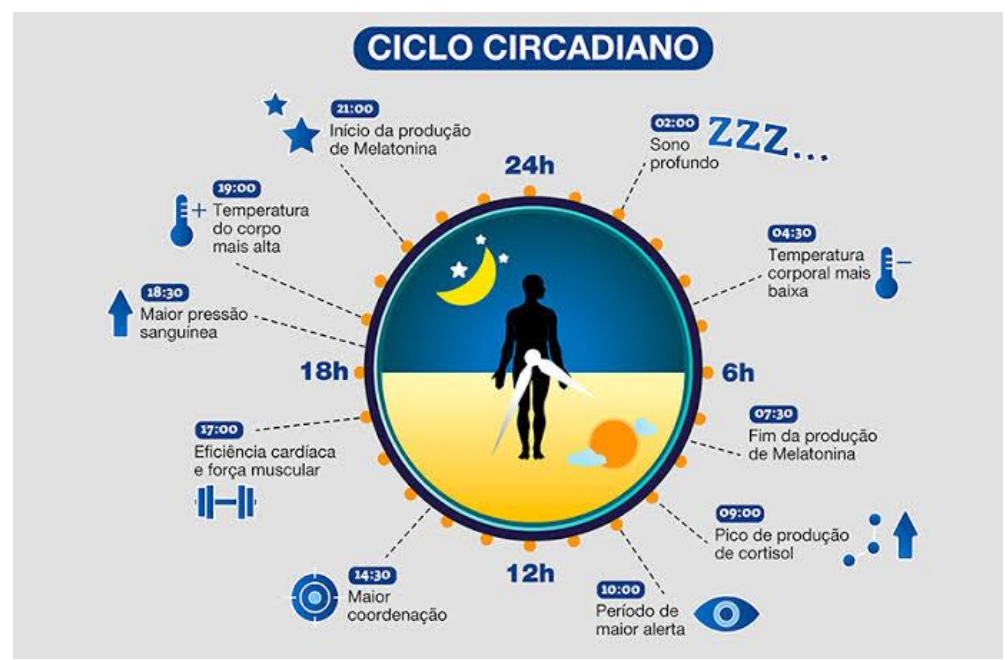

Fonte: Active Pharmaceutica

\section{SONO EM PACIENTES IDOSOS COM DEMÊNCIAS}

Atualmente existem cerca de 25 milhões de idosos com demência, o que constitui um sério problema de saúde pública. Em 2025 teremos cerca de 43 milhões de idosos nesta situação e a grande maioria (70\%) nos países em vias de desenvolvimento (Organização Mundial de Saúde). Gráfico B (www.who.int, 2008; www.alzheimerportugal.org, 2008).

Estima-se que a doença afete cerca de $5 \%$ da população mundial com idade compreendida entre 60 e 65 anos, $15 \%$ dos 65 aos 70 anos, uma em cada três pessoas entre os 80 e 85 anos e $50 \%$ acima dos 85 anos (Kaplan e Sadock, 2007).

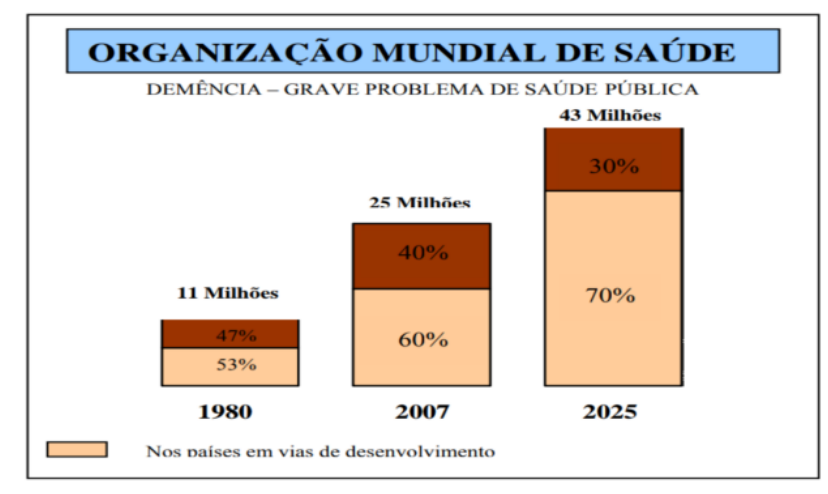

Gráfico 2- Número de indivíduos com demência

Fonte: A Perspectiva do Farmacêutico frente a doença de Alzheimer 
O Sono em pacientes idosos é fragmentado, ou seja, além da dificuldade em "pegar" no sono, acordam várias vezes durante a noite. Com a evolução das Demências os pacientes passam a apresentar com mais freqüência a SED, o que prejudica o sono noturno e trás agitação, nervosismo e mais confusão mental.

Tem um termo que se chama "Sundowning" que é o estado em que pacientes demenciados ficam mais agitados ainda, alterados, sem orientação, podem gritar e ficam violentos. A tendência é que essa situação ocorra mais a noite e devido a isto em conjunto com o uso de medicações, os idosos com Demências sofrem com muitos distúrbios do sono. Alguns tem insônias e praticamente não dormem, outros dormem porém ficam como se estivessem alucinando com gritos, tem visões, se alguém se aproxima são agressivos e ao despertar estão mais cansados pois não tiveram um sono reparador e sim um sono de vigilância.

Os Distúrbios do Sono em pacientes Demenciados são pouco conhecidos e muito confundidos com sintomas da própria doença levando ao uso de antipsicóticos. Assim temos a importância de analisar cada indivíduo, seus hábitos, suas doenças crônicas pré existentes pra que se consiga um tratamento ideal e uma boa qualidade de sono.

\section{O PAPEL DO FARMACÊUTICO NO ACOMPANHAMENTO DA FARMACOTERAPIA DE IDOSOS COM DEMÊNCIAS E SOLUÇÃO PRA DINÂMICA DE SONO EFICAZ}

Estima-se que 35 a $60 \%$ dos pacientes idosos estão expostos a potenciais interações medicamentosas, destas, 5 a 10\% evoluem para uma reação adversa grave, ou seja, reação nociva e desagradável, resultante de intervenção relacionada ao uso de um medicamento. Essas reações adversas podem predispor o idoso à ocorrência de diversos eventos adversos, como quedas, fraturas, confusão pós-operatória, sangramentos gastrointestinais, constipação, piora no quadro de insuficiência cardíaca congestiva, depressão, déficit cognitivo e disfunção renal. (Magro L, Moretti U, Leone R. Epidemiology and characteristics of adverse drug reactions caused by drugdrug interactions. Expert Opin Drug Saf. 2012 jan).

O acompanhamento farmacoterapêutico é um instrumento da Atenção Farmacêutica, no qual o farmacêutico se responsabiliza pelas necessidades do paciente relacionadas à farmacoterapia mediante a detecção, a prevenção e a resolução de problemas relacionados aos medicamentos. (MASCHIO DE LIMA, Tiago Aparecido et al. 2016). 
Particularmente entre os idosos, as desvantagens da automedicação devem ser consideradas e, entre elas, destacam-se os gastos desnecessários, atraso no diagnóstico e na terapêutica adequados, potenciais riscos de interações com os medicamentos prescritos, resistência bacteriana, reações adversas e intoxicação. (Sá MB, Barros JAC, Sá MPBO. Automedicação em idosos. Rev Bras Epidemiol 2007).

\begin{tabular}{|c|c|c|}
\hline Grupo de medicamentos & Drogas & Dose para adultos (mg) \\
\hline $\begin{array}{l}\text { Benzodiazepínicos de curta duraçáo } \\
\text { (vida média < } 10 \text { horas) }\end{array}$ & $\begin{array}{c}\text { Oxazepam } \\
\text { Triazolam } \\
\text { Alprazolam } \\
\text { Estazolam } \\
\text { Lorazepam } \\
\text { Temazepam }\end{array}$ & $\begin{array}{c}15-30 \\
0,125-0,25 \\
0,25-0,5 \\
0,5-2 \\
0,5-1 \\
15\end{array}$ \\
\hline \multirow[t]{2}{*}{$\begin{array}{l}\text { Benzodiazepínicos de longa duraçăo } \\
\text { (meia vida > } 20 \text { horas) }\end{array}$} & $\begin{array}{l}\text { Clordiazepóxido } \\
\text { Clonazepam } \\
\text { Diazepam } \\
\text { Flurazepam }\end{array}$ & $\begin{array}{c}5 \\
0,25-0,5 \\
2-10 \\
15-30\end{array}$ \\
\hline & Näo benzodiazepinicos & \\
\hline Agonista do receptor de melatonina & Ramelteon & 8 \\
\hline Pirazolopirimidina & Zaleplon & $5-10$ \\
\hline Imidazopiridinas & Zolpidem & $5-10$ \\
\hline \multirow[t]{2}{*}{ Ciclopirrolonas } & Zopiclone & - \\
\hline & Antidepressivos com efeitos sedativos & \\
\hline Triciclicos & $\begin{array}{l}\text { Amitriptilina } \\
\text { Imipramina }\end{array}$ & $\begin{array}{c}10-75 \\
25-100\end{array}$ \\
\hline Tetraciclico & Mirtazapina & $30-45$ \\
\hline Inibidor da recaptaçáo de serotonina & Trazodona & $50-150$ \\
\hline
\end{tabular}

Obs. Em pacientes idosos, iniciar com metade da dose média de adulto, e ajustar se necessário.

Fonte: Sono no envelhecimento normal e patológico: aspectos clínicos e fisiopatológicos.

$\mathrm{Na}$ tabela constam alguns medicamentos muito utilizados tanto no tratamento de Demências quanto nos distúrbios do sono. A classe mais famosa entre eles são os benzodiazepínicos. Benzodiazepínicos é uma classe de medicamentos hipnóticos sedativos utilizados para dormir e precisa de receita Azul Tipo B para adquiri-lo. Infelizmente por não se ter um tratamento específico pra perturbações de sono em idosos, esses medicamentos são muito recomendados por médicos mas tem efeitos colaterais como sonolência, baixa concentração, mudança de humor, agressividade e por fim insônia por rebote. Dessa forma é um ciclo vicioso onde tenta se tratar com medicamentos a Demência mas em contrapartida tem distúrbios do sono e quando tenta tratar os distúrbios do sono altera algo fisicamente que desperta um sintoma mais intenso da Demência. Por fim, não há um tratamento específico mas existem soluções simples pra minimizar essas alterações. 
Sendo assim, o tratamento dos distúrbios do sono em idosos antes de tudo deve ser baseado na estrutura fisiológica que é alterada com a idade. Padrões simples a serem seguidos diariamente já farão muita diferença no sono. Criar rotina de horários pra deitar-se e levantar; ter um ambiente com baixa luminosidade e pouco ruído; tomar banho de preferência morno pra relaxar e tomar sol na parte da manhã traz benefícios comprovados pro dono restaurador a noite. Tem também padrões a não serem seguidos: tomar café e bebida alcoólica perto da hora de dormir, cochilar a tarde durante muito tempo, ficar em ambiente muito movimentado onde não consiga relaxar.

\section{CONCLUSÃO}

Podemos observar o importante papel do farmacêutico ao paciente idoso, o acompanhamento e a orientação para a eficácia do seu tratamento farmacológico. Os Distúrbios do sono afetam a população em geral porém este trabalho teve o intuito de mostrar a realidade de grupo de idosos que possuem Demências como uma forma de alerta pois com a expectativa de vida aumentando, teremos muito mais idosos futuramente e conseqüentemente mais indivíduos necessitando de tratamento especializado pra se obter qualidade de sono e de vida.

\section{REFERÊNCIAS}

ANDRADE MA, Silva MVS, Freitas O. Assistência Farmacêutica como Estratégia para o Uso Racional de Medicamentos em Idosos. Semina Ciênc Biol Saúde 2004;25(I):55-63.

ARAÚJO, Rosana Soares; PONDÉ, Milena Pereira. Eficácia da memantina na doença de Alzheimer em seus estágios moderado a grave. Jornal Brasileiro de Psiquiatria, v. 55, p. I48-I53, 2006.

BOTtinO, Cássio Machado de Campos. O tratamento de longo prazo está indicado para pacientes com doença de Alzheimer?. 2005.

Brasil Ministério da Saúde. Atenção a saúde do idoso: instabilidade postural e queda. Brasil: MS, 2000.

CAMARGOSi, E. F. et al. Incidência de distúrbios do sono em pacientes com doença de Alzheimer. Einstein, Brasília, v. I, n. II, p. 46i-465, nov./20Ir.L.L.C.

CASTRO,Evolução da pesquisa em atenção farmacêutica no Brasil: Um estudo descritivo I9992003,2006 
CFF. Conselho Federal de Farmácia. Dados. 2019. Disponível em: https://www.crfms.org.br/noticias/farmaceutico/silg-no-mes-do-uso-racional-demedicamentos-conselho-de-farmacia-alerta-para-perigo-da-automedicacao-napandemia\#: :text=A\%2opesquisa\%2oconstatou\%20ainda\%20que,menos\%20uma\%20vez\%2opor\% 20semana. Acesso em o6 Set 2021.

CHRISTOFOLETTI[A], [. G. et al. Locomoção, distúrbios neuropsiquiátricos e alterações do sono de pacientes com demência e seus cuidadores. Fisioter, Curitiba, v. 26, n. I, p. 47-53, mar./2013. E. F. et al. Incidência de distúrbios do sono em pacientes com doença de Alzheimer. Einstein, Brasília, v. I, n. II, p. 46I-465, nov./2oir.

FERNANDES, W. S.; CEMBRANELLI, J. C. Automedicação e o uso irracional de medicamentos: o papel do profissional farmacêutico no combate a essas práticas. Revista Univap, v. 21, n. 37, p. 5-12, 2015 .

GOMES2, M. S. Q. ;. M. D. M. Sono no envelhecimento normal e patológico: aspectos clínicos e fisiopatológicos. Rev Bras Neurol, , Botafogo, v. 47, n. I, p. 31-42, mai./2021.

LEITE, J. C. O. R. M. A Perspectiva do Farmacêutico na Doença de Alzheimer. I. ed. Porto: [s.n.], 2008. p. I-67.

LIMA, T. A. M. et al. Acompanhamento farmacoterapêutico em idosos. Revista Arquivos de Ciências da Saúde, v. 23, n. I, p. 52-57, 2016.

MEDSCAPE. Privação de sono aumenta nível plasmático de biomarcador de Alzheimer. Disponível em:https://portugues.medscape.com/verartigo/6503719. Acesso em: I4 set. 2021.

MENESES A L L, Sá M L B. Atenção farmacêutica ao idoso: fundamentos e propostas. Geriatria \& Gerontologia. 2010;4(3):154-161.

PEBMED. Sono e o Envelhecimento. Disponível em: https://www.google.com/amp/s/pebmed.com.br/sono-e-envelhecimento-parte-I-alteracoesfisiologicas-e-abordagem-geral-dos-disturbios-de-sono-em-idosos/. Acesso em: I2 ago. 2021. RELVAS, J . (2006). Psicofarmacologia das pessoas idosas, In H. Firmino. Psicogeriatria. Coimbra: Psiquiatria Clínica.

RIBEIRO AQ. ROZEN FELD, KLEINCH,CÉSAR RC C, ACURCIO FA .Inquerito sobre uso de medicamentos por idosos aposentados, Belo Horizonte, MG .REV Saúde Pública; V.42 p: 72432,2012 . 
SANTOS et. al., FC Santos - Arquivos brasileiros de..., de 2017-Scielo Brasil.

SÁ2, A. L. L. D. M. M. L. B. Atenção farmacêutica ao idoso: fundamentos e propostas. Sociedade Brasileira de Geriatria e Gerontologia, Ceará, v. 4, n. I, p. ı-9, out./2oı.

SALES, Alessandra Santos, SALES CEZAR, Marta, CASOTTI ,Gabriele Santos Augusto. Perfil farmacoterapêutico e fatores associados à polifarmácia entre idosos de Aiquara, Bahia, em 2014 Epidemiol. Serv. Saúde 26 (oI) JanMar2017•https://doi.org/10.5123/Si679-4974201700o10oor3 SERENIKI, Adriana; VITAL, Maria Aparecida Barbato Frazão. A doença de Alzheimer: aspectos fisiopatológicos e farmacológicos. Revista de psiquiatria do Rio Grande do Sul, v. 30, 2008.

TAUB, Anita; ANDREOLI, Sergio B.; BERTOLUCCI, Paulo H. Dementia caregiver burden: reliability of the Brazilian

version of the Zarit caregiver burden interview. Cadernos de Saúde Pública, v. 20, p. 372-376, 2004. VIEIRA, F; S. Possibilidade de contribuição do farmacêutico para a promoção de saúde. Revista ciências e saúde coletiva. V.I2, n.I, Rio de Janeiro jan-mar 2007.

WILLIAN REZENDE. Conheça a Relação entre o sono e Demências. Disponível em: https://www.willianrezende.com.br/relacao-entre-sono-e-demencia/. Acesso em: I5 set. 202I.

XAVIER $_{3}$, J. M. N. D. S. ;. A. C. M. C. ;. W. W. M. ;. C. L. Avaliação da qualidade de sono em idosos não institucionalizados. ConScientiae Saúde, 2012;1I(I):29-36., Teresina, v. II, n. I2940, p. 2936 , nov./20II. 